\title{
A Method for Determination of the Timing Stability of PET Scanners
}

\author{
Christopher J. Thompson*, Member, IEEE, and Andrew L. Goertzen
}

\begin{abstract}
We report on the timing resolution and stability of the MicroPET R4 PET scanner. Its detectors have energy resolutions in the range of $25 \%$ and previously reported timing resolutions $3.2 \mathrm{~ns}$. Our preliminary evaluation of this instrument showed that artefact-free normalization sinograms could only be obtained with a timing window of $10 \mathrm{~ns}$ or more in spite of a timing resolution of 3.2 ns. This instrument uses high-speed electronics albeit with 2 -ns timing clock. We performed sham transmission scans with nothing in the field of view, and a range of timing windows from 2 to 14 ns and used a 14-ns timing blank scan to generate effective attenuation sinograms as a function of timing window. These showed trues count-rates which fit well to a ERF $(\tau)$ function. However, the effective attenuation value, which should be 1.0, changes from block to block and becomes very high in some blocks ( $>3.5$ at $6 \mathrm{~ns}$ ) suggesting the need for timing alignment. A method was devised to measure the timing stability to a precision better than the timing bin width of 2 ns.
\end{abstract}

Index Terms-Positron emission tomography, timing alignment, timing stability, time-of-flight.

\section{INTRODUCTION}

$\mathbf{P}$ OSITRON emission tomography (PET) scanners using lutetium oxy-orthosilicate (LSO) crystals should have better performance at high count-rates than those with bismuth germanate (BGO) crystals. LSO has much more light output and a faster decay time (40 ns) than BGO (300 ns). One would expect that LSO-based scanners would use a narrower timing window than those with BGO detectors. Whole-body BGO scanners (like the CTI ECAT HR+ scanner installed at the Montreal Neurological Institute in 1997) typically use a timing window of $14 \mathrm{~ns}$. We recently acquired a MicroPET R4 [1] (CTI Concorde Microsystems, Knoxville, TN), which uses LSO crystals, and allows timing windows of 2, 6, 10, and $14 \mathrm{~ns}$.

The first author has recently developed a time-alignment probe [2], [3] for PET scanners. This uses a central positron-emitting source embedded in a plastic scintillator to which all the detectors in a PET scanner can be aligned by placing the probe at the center of the scanner and recording

Manuscript received November 12, 2004; revised May 12, 2005. This work was supported in part by a grant from the Canadian Foundation for Innovation. The work of C. J. Thompson was supported by the Natural Science and Engineering Council of Canada under Grant OGP-0036672. The Associate Editor responsible for coordinating the review of this paper and recommending it for publication was D. Townsend. Asterisk indicates corresponding author.

*C. J. Thompson is with the Montreal Neurological Institute, 3801 University St. \#798, Montreal, QC H3A 2B4, Canada (e-mail: christopher.thompson@mcgill.ca).

A. L. Goertzen is with the Montreal Neurological Institute, Montreal, QC H3A 2B4, Canada (e-mail: goertzen@bic.mni.mcgill.ca).

Digital Object Identifier 10.1109/TMI.2005.852072 the delay between the emission of the positron and the detection of the gamma ray. This is expected to allow the timing alignment to be performed much faster and more accurately than previously. Several of these probes have been produced by Scanwell Systems (Montreal, QC, Canada) and an initial evaluation of their performance was presented at the IEEE MIC 2004 conference [3]. We were interested to find out how stable the timing alignment of a modern PET scanner was over time. This could be done by positioning the time alignment probe on the couch such that the source was in the center of the scanner. Unfortunately, signals from the timing probe cannot be introduced into the coincidence processor of any current scanner, so it is not possible to measure the timing stability with the timing probe. Therefore, an indirect method, involving a very narrow timing window and mock transmission scans, was devised to investigate the timing alignment and stability.

\section{Materials AND MethodS}

The experiments were performed on a MicroPET R4 PET scanner designed for imaging small rodents. This scanner has sophisticated software for performing the crystal identification and energy window setup. However, unlike most PET instruments [4], [5], there was no provision for the timing offsets of each detector to be adjusted by the end-user at the time the scanner was delivered. (This has been corrected in version 5.2.2.8 of the MicroPET software.) It is also not possible to access the signals from each detector to measure the timing spectra, although the timing resolution has been reported as 3.2 ns [6]. In this scanner, the arrival of a gamma ray is assigned a time with respect to a clock with an effective frequency 500 MHz. The coincidence circuit can accept events which have identical time stamps or differ by 1,2 , or 3 ( 2 ns) clock ticks. There is an inherent uncertainty of $1 / 2$ of a clock tick in the effective width of the timing window. This gives effective timing windows $(2 \tau+1)$ of $3,7,11$, and $15 \mathrm{~ns}$. Since the MicroPET software refers to these timing windows as in terms of integral multiples of the clock period, we will use the window width nomenclature of $2,6,10,14$ ns to be consistent with the instrument's user interface.

\section{A. Experimental Procedures}

The scanner has a single ${ }^{68} \mathrm{Ge}$ point source for performing transmission scans. It performs a spiral orbit around the field of view with a period of $516 \mathrm{~s}$. In order to assess the timing alignment, we used the scanner's transmission scanning point source and acquired one blank scan with a $14 \mathrm{~ns}$ timing window 
for $5160 \mathrm{~s}$ (i.e., ten passes through the scanner). We then acquired sham "transmission scans" (with nothing in the field of view) using a 2, 6, 10, and 14 ns window also for $5160 \mathrm{~s}$. These studies were acquired within a two day period. Then over the next week we acquired four more scans with a 2-ns window. A blank scan and a transmission scan acquired with identical scan and resolving times should be equivalent. However, in the MicroPET software, the protocols have a different user interface, since when one processes a transmission scan, a blank scan file name is required in order to calculate the attenuation sinogram.

In July 2004, the scanner was returned to the manufacturer after an explosion and fire in an adjacent room caused a significant accumulation of soot inside it. After cleaning and rebuilding the scanner, the software was upgraded and provision is now made to perform a detector timing alignment on each crystal element to an accuracy of one clock tick (2 ns). The series of experiments with various timing windows was then repeated. We then did 12 more trials of the 2-ns window study and in addition we added trials during which the room temperature was raised (trial \#10) and lowered (trial \#12) by $2{ }^{\circ} \mathrm{C}$ for $1 \mathrm{~h}$ preceding the scan, and for the entire scan duration. This was done to ascertain whether the time alignment was sensitive to temperature fluctuations and to simulate the drift which could occur over time in the alignment.

\section{B. Data Analysis}

The total prompt and delayed counts were extracted from the file headers of all studies. The prompt-delayed and delayed counts were plotted as a function of the timing window width for both studies. We estimated the global timing resolution as follows. The full width at half maximum (FWHM) of a Gaussian curve is related to its standard deviation by

$$
\mathrm{FWHM}=2 \sigma \sqrt{2} \ln 2 \quad \mathrm{FWHM}=2.35 \sigma .
$$

The true counts, $T_{\text {window }}$, acquired in a timing window of width $W$ ns is equivalent to integrating a Gaussian whose area is the total true counts, $\mathrm{T}_{\text {total }}$, over the timing window, so that

$$
\mathrm{T}_{\text {window }}=\mathrm{T}_{\text {total }} \operatorname{erf}(2.35[\mathrm{~W} / \mathrm{FWHM}]) \text {. }
$$

Knowing the counts acquired in each timing window, $\mathrm{T}_{\text {window }}$ the FWHM was estimated by fitting this equation from data obtained with the four effective timing windows, $(3,7,11,15)$ and the counts obtained in both data sets using Graph-PAD Prism Version 4.0 [7].

The delayed counts reported in each study as a function of the resolving time window was used to fit the slope of the randoms versus timing window width in the early and late scans. This slope should be proportional to the source activity if the dead time in each study was the same. Since the MicroPET software dese not report dead time in transmission scans, this was used to get an independent measure of the dead time.

Sinograms of the effective attenuation of air measured by all crystal pairs were obtained using the manufacturer's histogram tool in the way it is normally used to create an attenuation map to correct emission images. Ideally these sinograms should all contain numbers which are very close to 1.0 , since there should be no attenuation measured. The timing window represents a
Windowed Timing Spectrum

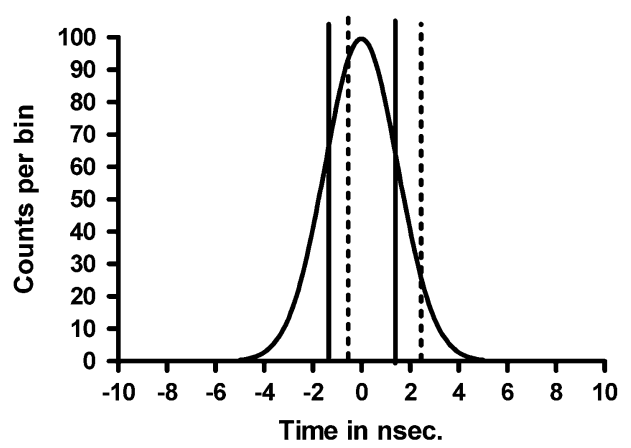

Fig. 1. Theoretical timing spectrum with a 3.5-ns FWHM. Two timing windows whose widths are $2.5 \mathrm{~ns}$ are shown overlaid on it. One is well aligned (solid lines) and another is misaligned by 2 ns (dashed lines). Only the region within the window contributes events to an image so there are fewer counts if the timing window is misaligned.

sample of the Gaussian timing spectrum from each detector pair. In practice, one would expect that the sinograms collected with the narrower windows, would have numbers somewhat greater than 1.0, and that detectors which were not correctly aligned to have values much greater than 1.0. Typical sinograms were selected from each data set. A region of interest encompassing an area which was quite uniform and another with an abnormally high attenuation coefficient were chosen for further study. The same ROIs were reused on each data set.

The apparent attenuation of the two regions was then plotted as a function of resolving time. (Different regions were chosen for both the first and second studies.)

\section{Estimation of Timing Drift}

The 2-ns timing window takes a sample of the timing spectrum of all coincident detector pairs. It is well known that there are variations in the apparent detection time of gamma rays by PET detectors which must be accounted for. The 2-ns window is significantly narrower than the FWHM of the timing spectrum. If two detectors are correctly time-aligned, this window will be near the "peak" of the timing spectrum. If they are misaligned then this window will be on the sloping portion of the timing spectrum. This is illustrated in Fig. 1. The solid vertical lines represent the limits of a well aligned timing window, while the dashed lines represent a timing window which is misaligned by about 2 ns. We know that the window is always 2 ns. We assume that all detectors have the same timing spectrum. From our experiments we determine the range of values taken on by a badly aligned detector. We can use this to make an estimate of the timing drift which would have to be present to cause the observed changes in apparent attenuation. Since we are basing this estimate on the ratio of two scans done with different timing windows, this cancels out any detector-to-detector efficiency variations.

If the timing window is offset, as is shown in Fig. 1, fewer true counts are detected. We estimated the effective timing window offset which would be required to produce a certain fractional loss of true counts. This was done by integrating a Gaussian whose FWHM matched that determined in the previous section, and then displaced in steps of $0.1 \mathrm{~ns}$ up to a maximum 


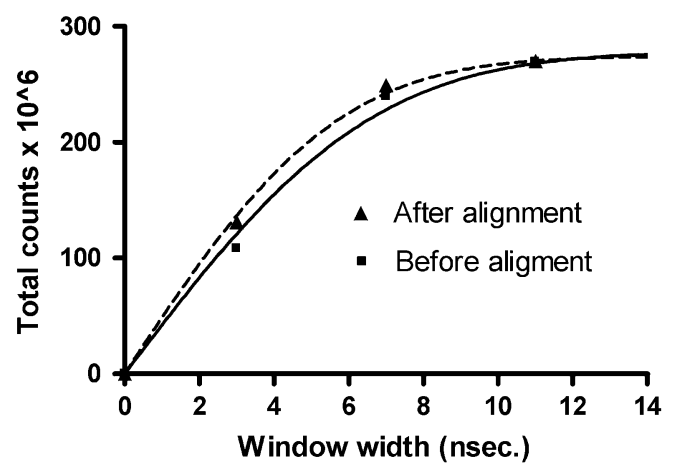

Fig. 2. Total true + scattered counts acquired as a function of the timing window width during two series of experiments performed 6 months apart (shown on the graph as "Before" and "After alignment"). The data points are fitted to (2) and shown as a solid line (before), and dashed line (after). They are normalized to the same value at 14-ns timing window in order to demonstrate the improved timing alignment.

TABLE I

FitTing Results SHOWING Relative TRue COINCIDENCE EFFICIENCY, FWHM AND THE SLOPE OF THE RANDOMS VERSUS WINDOW WIDTH

\begin{tabular}{c|c|c|c}
\hline & Trues & Trues & Randoms \\
\hline $\begin{array}{c}\text { Study } \\
\text { (align) }\end{array}$ & $\begin{array}{c}\text { Plateau } \\
\text { (MC) }\end{array}$ & $\begin{array}{c}\text { FWHM } \\
\text { (nsec) }\end{array}$ & $\begin{array}{c}\text { Slope } \\
\text { (MC/nsec) }\end{array}$ \\
\hline Before & 272 & $3.13 \pm .12$ & 0.673 \\
\hline After & 243 & $2.68 \pm .28$ & 0.373 \\
\hline
\end{tabular}

timing error of $5 \mathrm{~ns}$. The ratio of the area under this function, integrated from -7.0 to $+7.0 \mathrm{~ns}$, to that obtained for each value of time skew, was plotted against the time skew for each timing window. The vertical axis of these data represents the apparent attenuation for a given time skew and window width, so that the change in apparent attenuation from day to day can be interpreted as timing drift.

\section{RESULTS}

Fig. 2 shows the true counts acquired over the duration of the two sets of experiments before and after the timing alignment was performed. The trues were fitted to an error function according to (2). The figure shows the data normalized to the peak counts obtained in a 14-ns window in order to demonstrate the more rapid approach of the counts to their peak value with a wide timing window. The parameters of the fits are shown in Table I. The plateau of the fit to the true counts is in mega-counts (MC), and the slope of the random counts is in MC/ns of timing window. The two series of studies were done 183 days apart during which the ${ }^{68} \mathrm{Ge}$ source had decayed to $67 \%$ of is prior activity. The plateau reached as the timing window increases changed from 273 in the first study to 243 million counts in $5160 \mathrm{~s}$. The ratio of the total counts in the second to that of the first study is 0.89 so that the scanner's efficiency has apparently increased by a factor of $0.89 / 0.67$ or 1.33 as a result of the improved time alignment. Some of this is due to reduced dead-time, but the scanner's software does not report dead-time for transmission scans. Randoms rates were reduced to $55 \%$, instead of $65 \%$ based on the source's decay. The change in dead time during transmission scans should be $(65 / 55-1)$ or $20 \%$.
Fig. 3 shows the sinogram chosen for further study during the first series of experiments. This portrays the effective attenuation of air with different timing windows. The lighter regions indicate detectors whose timing spectra are poorly resolved, or are not in correct time alignment with other detectors. The grayscale on these studies saturates with peak white at an effective attenuation value of 5.0 and has a minimum value of 1.0. One can see that as the resolving time is shortened, the images get brighter. There are some diagonal bands which become much brighter indicating an increase in apparent attenuation. These correspond to detectors which are mis-aligned. When a whole block is misaligned, the band is eight crystals wide. There are narrow lines which correspond to only a single crystal in a block being misaligned. The ROIs chosen for further study are visible on these sinograms as rectangular outlines.

Fig. 4 shows the effective attenuation as a function of resolving time for the two regions as a function of time. In the left panel (done before the timing alignment was possible), the regions chosen are those identified in Fig. 3. In the right panel the scales are different since the attenuation factors were much lower. Not only are the mean values lower, the consistency within the region is improved as indicated by the reduced-error bars.

Fig. 5 represents the theoretical change in apparent attenuation factor which would result from a timing mis-alignment of up to $3.0 \mathrm{~ns}$ for the range of timing windows allowed on this scanner. By locating the various attenuation factors plotted in Fig. 4 on the vertical axis of Fig. 5, one can estimate what degree of timing skew would cause the changes observed during these experiments. (This was done by drawing a thin-walled rectangle on the original image of Fig. 5 in GraphPad Prism. The $X, Y$ coordinates of one corner of the rectangle are then displayed as numbers in Prism, and the thin vertical and horizontal lines representing the outer boundary of this rectangle are shown in Fig. 5.) Using typical values from the second experiment, a change from 3.0 to 3.3 in attenuation factor corresponds to a timing drift of $370 \mathrm{ps}$. In the first experiment the changes in apparent attenuation are much greater since the timing is badly aligned, but the maximum variation corresponds to less than 300 ps drift.

During the tenth and twelfth measurements of the second series, the room temperature was raised and lowered by $2^{\circ} \mathrm{C}$. The results from these runs were within the range of the day-to-day fluctuations during which the temperature remained constant, so there appears to be no observable timing drift with modest temperature change.

\section{DISCUSSION}

Our early experience with this scanner suggested that the use of a timing window shorter than $10 \mathrm{~ns}$ would cause a significant loss of sensitivity in certain lines of response. It appears that this is due to either poor timing resolution, or incorrect time alignment. Since the time alignment between detectors could not be adjusted with the tools provided, and the timing spectrum cannot be measured, it was not possible to determine which is to blame. The second set of experiments, made after the manufacturer provided a procedure for timing alignment, show much better 


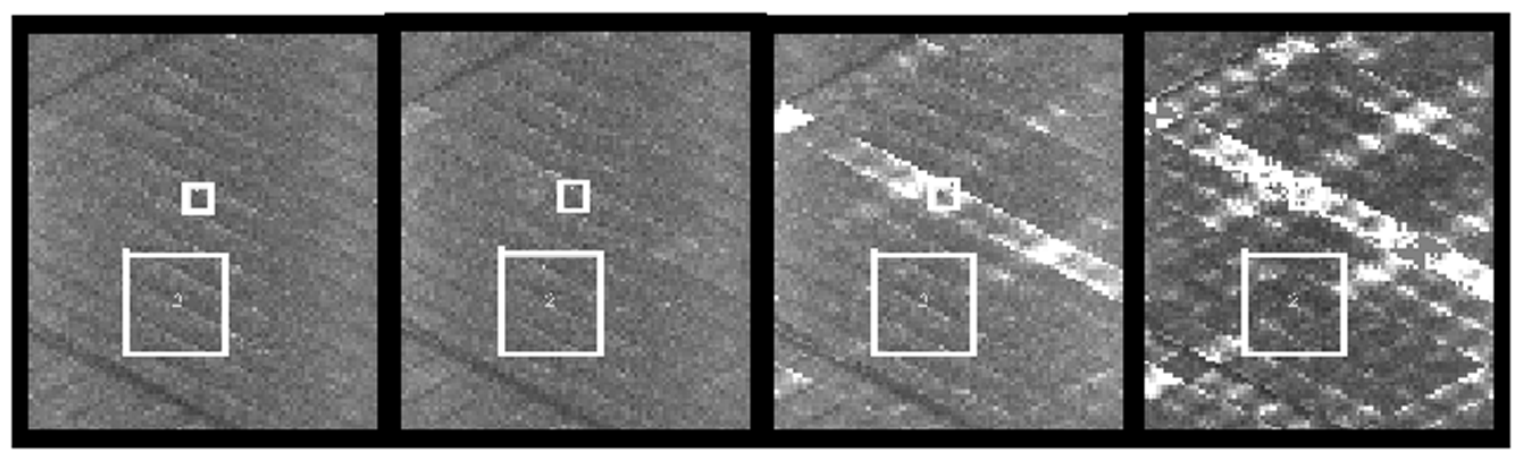

Fig. 3. Attenuation sinograms histogrammed using a 14 (far left), 10, 6, and 2 (right) coincidence window as the transmission scan, and a 14-ns window for the blank scan. The two square ROIs were used for the stability analysis in the first set of experiments.

\section{Before timing alignment}

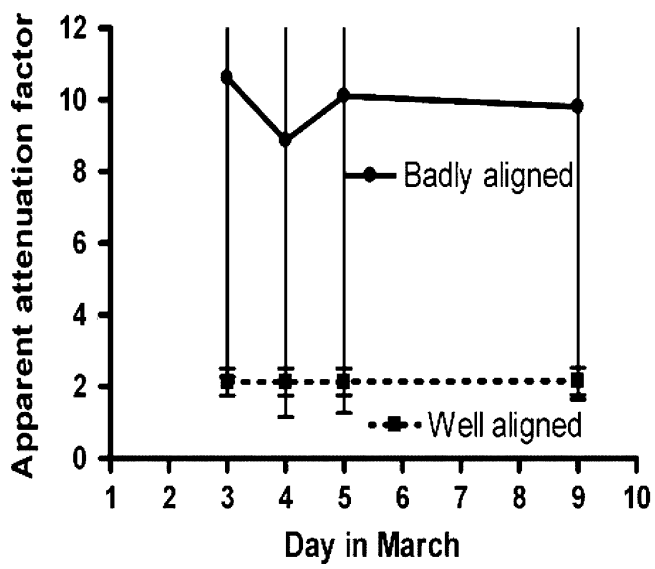

After timing alignment

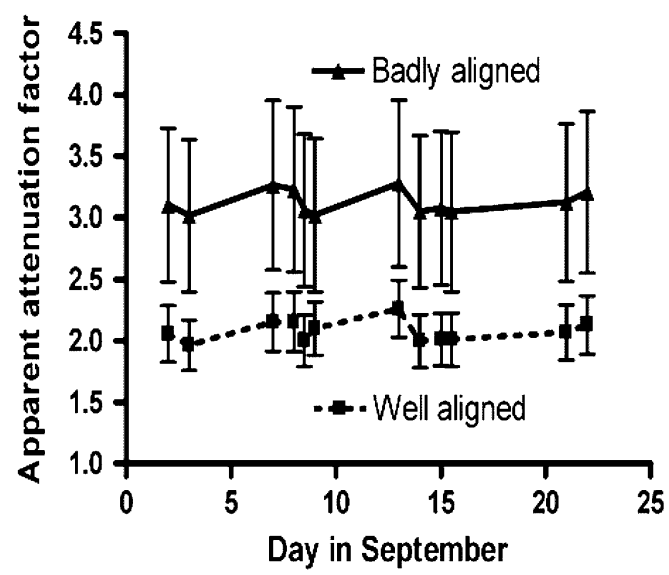

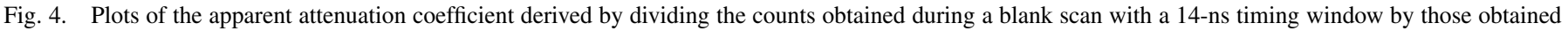

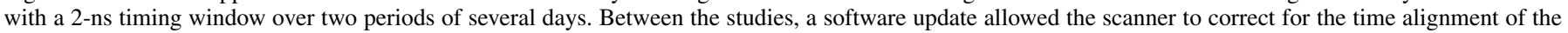
detectors. Note the different vertical scales for each study.

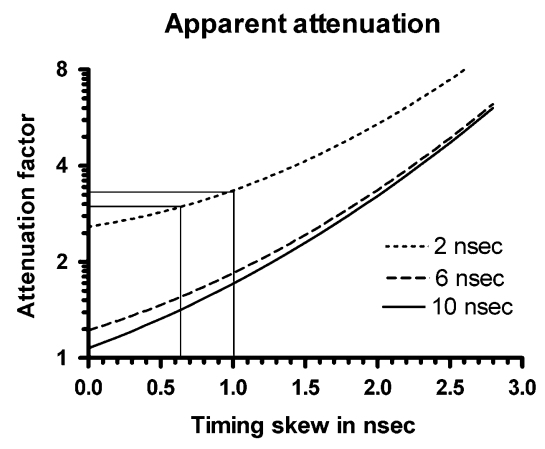

Fig. 5. Apparent attenuation factor which would be obtained if a timing window of various widths were used to sample a Gaussian timing spectrum whose FWHM was $3.3 \mathrm{~ns}$ if the timing alignment were skewed by up to $3 \mathrm{~ns}$.

timing alignment. There are no block-detector streaks in the 6-ns attenuation sinograms after the alignment was performed. One could anticipate that henceforth there will be no artefacts introduced into transmission scans using a 6-ns window. No significant drift was observed in the second series of experiments with the 2-ns window. Since this window is narrower than the FWHM of the timing spectrum, there is a significant loss of prompt counts (efficiency) with this timing window, and it should not be used in practice.
The original purpose of these experiments was to determine how often the performance of a timing alignment might be required on a scanner capable of time-of-flight PET. In a companion paper [3], we report on a new technique for doing the timing alignment of PET scanners. Given the timing resolution of the present scanners (2-3 ns), the timing instability measured in this work (few hundred ps) is negligible. However, time-offlight (TOF) scanners with better time resolution are now being introduced [8]. A timing alignment probe for day-to-day alignment could be useful as part of the quality control procedure. Much more accurate time alignment would be required if time of arrival differences of less than $1 \mathrm{~ns}$ were to be used to estimate the location of the positron along a line of response.

The time digitization into 2-ns bins as used in the MicroPET is not adequate to determine the timing stability with the required accuracy. Recently, more accurate methods have been introduced for measuring smaller time differences [9] using time to voltage converters. However, estimating the effective attenuation along each LOR with different values in a blank and transmission scan provided the tools required to make a more precise estimate of the timing drift. In both the first and second series of experiments, the drift appears to be less that $400 \mathrm{ps}$. This corresponds to a gamma ray's time-of-flight distance of $12 \mathrm{~cm}$. (Or a TOF positioning error of $6 \mathrm{~cm}$.) The range of values among both 
the good and bad detectors in both studies is 200 to 370 ps. This suggests that the timing stability of a PET scanner with detectors and modern readout electronics could be stable enough to operate in a time-of-flight mode without requiring daily timing alignment.

\section{REFERENCES}

[1] C. Knoess, S. Siegel, A. Smith, D. Newport, N. Richerzhagen, A. Winkeler, A. Jacobs, R. N. Goble, R. Graf, K. Wienhard, and W. D. Heiss, "Performance evaluation of the MicroPET R4 PET scanner for rodents," Eur. J. Nucl. Med. Mol. Imag., vol. 30, no. 5, pp. 737-747, 2003.

[2] C. J. Thompson and M.-L. Camborde, "An Instrument and Method to Facilitate and Improve the Timing Alignment of a PET Scanner," U.S. Patent pending, Nov. 2002.
[3] C. J. Thompson, M.-L. Camborde, and M. Casey, "A central positron source to perform the timing alignment of detectors in a PET scanner,' IEEE Trans. Nucl. Sci., vol. 52, no. 4, 2005, to be published.

[4] D. Luo, J. J. Williams, M. K. Limkeman, M. J. Cook, E. L. Oswalt, and D. L. McDaniel, "Crystal-based coincidence timing calibration for PET scanner," in IEEE MIC Conf. Rec. CD, vol. M11, 2002, p. 060.

[5] M. W. Lennox, Z. Burbar, J. Young, T. Gremillion, and C. Knoss, "Coincidence time alignment of high resolution planar detectors," in IEEE MIC Conf. Rec., vol. M6, vol. 2002, CD-ROM, p. 47.

[6] C. Tai, A. Chatziioannou, S. Siegel, J. Young, D. Newport, R. N. Goble, R. E. Nutt, and S. R. Cherry, "Performance evaluation of the microPET P4: A PET system dedicated to animal imaging," Phys. Med. Biol., vol. 46 , no. 7, pp. 1845-1862, 2001.

[7] (2003) GraphPad Prism Version 4.00 for Windows. [Online]. Available: http://www.graphpad.com

[8] M. Conti, M. E. Casey, and F. Kehren, "Implementation of time-of-flight on CPS HiRez PET scanner," presented at the IEEE Medical Imaging Conf., Rome, Italy, Oct. 2004.

[9] B. K. Swann et al., "A 100-ps time-resolution CMOS time-to-digital converter for positron emission tomography imaging applications," IEEE J. Solid State Circuits, vol. 9, no. 11, pp. 1839-1852, Nov. 2004. 